\title{
Role of anti-mullerian hormone as a diagnostic tool for polycystic ovary syndrome
}

\author{
Shakun Singh, Arshiya Firdaus*, Rachna Chaudhary, Vandana Dhama
}

Department of Obstetrics and Gynecology, LLRM Medical College, Meerut, Uttar Pradesh, India

Received: 24 June 2020

Accepted: 31 July 2020

\section{*Correspondence:}

Dr. Arshiya Firdaus,

E-mail: arshi2210@gmail.com

Copyright: (C) the author(s), publisher and licensee Medip Academy. This is an open-access article distributed under the terms of the Creative Commons Attribution Non-Commercial License, which permits unrestricted non-commercial use, distribution, and reproduction in any medium, provided the original work is properly cited.

\section{ABSTRACT}

Background: PCOS has been recognized as the most frequent endocrinopathy among reproductive aged women. This study has been done to determine whether the measurement of serum AMH can be used to diagnose PCOS and as a tool to predict the prognosis of PCOS.

Methods: This was a prospective case control study on a woman attending gynae OPD of LLRM Medical College, Meerut, from May 2018 to June 2019. Study comprised of 50 women diagnosed with PCOS using Rotterdam criteria and 50 controls. Clinical data were collected including history, oligomenorrhea, hirsutism, examination included BMI, FG score and blood investigations including serum FSH, LH, TSH, prolactin, estradiol and serum AMH level. USG was done for all women.

Results: Both PCOS cases and controls were matched for age and BMI. Mean level of AMH in PCOS cases and controls was 7.1096 and 2.423 respectively, AMH was two to three times higher in women with PCOS than controls which was statistically significant $(\mathrm{p}<0.05)$. Most frequent phenotype of PCOS in this study was phenotype A (48\%). Highest mean level of AMH was also found in phenotype A (OA+HA+PCOM), thus reflecting the severity of PCOS. Maximum diagnostic potential for PCOS was at cut off $4.22 \mathrm{ng} / \mathrm{ml}$ with sensitivity of $92.5 \%$ and specificity of $100 \%$. Conclusions: AMH level can be used as diagnostic and prognostic modalities in PCOS. AMH value rises when hyperandrogenism is present therefore serum AMH levels also reflect the phenotype of PCOS and severity of PCOS.

Keywords: Anti-mullerian hormone, Hyperandrogenism, Oligomenorrhea, Polycystic morphology, Polycystic ovarian syndrome, Rotterdam criteria

\section{INTRODUCTION}

Polycystic ovary syndrome (PCOS) also known as Stein Leventhal syndrome is one of the most common endocrine abnormalities among premenopausal women, yet its diagnosis remains one of the most challenging issues in endocrinology and reproductive medicine. ${ }^{1}$ It has been recognized as the most frequent endocrinopathy among reproductive-aged women, with a prevalence of 6$10 \%$ world-wide. $^{2}$ There is a recent rise in PCOS cases in urban India because of westernization, modernization, stress and life style changes. It is a complex heterogenous multisystem condition characterized by elevated androgen levels, menstrual irregularities, and/or small cysts on one or both ovaries. ${ }^{3}$ The disorder can be morphological (polycystic ovaries) or predominantly biochemical (hyperandrogenaemia). Hyperandrogenism, a clinical hallmark of PCOS, can cause inhibition of follicular development, microcysts in the ovaries, anovulation, and menstrual changes. ${ }^{4}$

It can present with wide spectrum of clinical features and delayed squeal like type 2 diabetes mellitus, cardiovascular diseases, metabolic syndrome and endometrial cancer which are preventable. ${ }^{4}$ It is caused by imbalance of sex hormones that leads to menstrual irregularities, infertility, anovulation and other metabolic disturbances. $^{5}$ 
Table 1: Diagnostic tools for polycystic ovarian syndrome.

\begin{tabular}{|lll|}
\hline NICHID/NIH criteria (1990) & $\begin{array}{l}\text { ESHRE/ASRM Rotterdam } \\
\text { criteria (2003) }\end{array}$ & $\begin{array}{l}\text { Androgen excess society (AES) criteria } \\
(2006)\end{array}$ \\
\hline Hyperandrogenism & Hyperandrogenism & Hyperandrogenism \\
\hline Oligo-ovulation/anovulation & Oligo-ovulation /anovulation & Ooligo-ovulation/anovulation \\
\hline Exclusion of other related disorders & Polycystic ovaries on ultrasound & Polycystic ovaries on USG \\
\hline & & Exclusion of others related disorders \\
\hline
\end{tabular}

In 2003, the Rotterdam consensus included PCOM finding as a criterion, and defined that the presence of at least two out of the three main characteristics were necessary to confirm the diagnosis (Table 1).

Therefore, the Rotterdam consensus expanded the possibilities of combinations of the three classic manifestations, allowing the characterization of four main phenotypes.

Rotterdam criteria although worldwide accepted has following drawbacks with respect to PCOM criteria:

1. Majority of PCOS are young obese females where TAS is difficult and TVS not possible as most of them are teenagers who are virgin

2. Assessment of AFC is subjective and not standardized with interobserver variability ${ }^{6}$

3. Phase of menstrual cycle and oral contraceptive use alter polycystic ovarian morphology and

4. Technical advances in imaging have led to an artificial increase in PCOM resulting in confusion over its use as a diagnostic criteria ${ }^{7-9}$

5. Diagnosis of hyperandrogenism is problematic clinically as calculation of Ferriman-Gallwey score is also subjective.

These diagnostic challenges emphasize the need for objective and quantitative diagnostic measures to guide in diagnosis and treatment of PCOS. Due to limitations associated in Rotterdam criteria, new tool AMH can be used as a potential objective, quantitative and biological diagnostic marker for PCOS.

Anti-mullerian hormone (AMH) has a glycoprotein dimer structure and is a member of the transforming growth factor- $\beta$ (TGF- $\beta$ ) family. The gene which encodes for AMH is localized on the small arm of chromosome. ${ }^{19}$ $\mathrm{AMH}$ in the female is produced exclusively by ovarian granulosa cells surrounding preantral and antral follicles, its concentration declines with age and become undetectable after menopause. The concentration of this hormone slightly fluctuates during different phases of the menstrual cycle but not significantly enough to affect its measurement and its level do not get altered due to oral contraceptive pills usage.

During human folliculogenesis, AMH protein expression begins at the primary follicle stage, highest expression is detected in FSH-dependent pre-antral and small antral follicles of $\leq 4 \mathrm{~mm}$ in diameter, and $\mathrm{AMH}$ expression gradually declines in subsequent stages and is absent in follicles larger than $8 \mathrm{~mm}$.

$\mathrm{AMH}$ has an important role in the development and maturation of follicles. ${ }^{10}$ Higher AMH levels in PCOS patients turns the follicles more resistant to FSH action, culminating in inhibition of follicular maturation and ovulation, and in inhibition of aromatase expression, and consequently, leading to hyperandrogenism.

\section{METHODS}

This was a prospective case control study conducted among the patients visiting the obstetrics and gynecology outpatient department of LLRM Medical College, Meerut during a period from May 2018 to June 2019.

Study comprised of 50 women with PCOS, diagnosed using Rotterdam criteria and 50 women as controls. Cases were women who met the diagnostic criteria for PCOS. Subjects who received hormonal therapy within three months before the beginning of the study were excluded.

Diagnosis of PCOS was established based on Rotterdam 2003 consensus, which is the finding of 2 out of the 3 following criteria:

- $\quad$ Oligo and/or anovulation;

- Hyperandrogenism, defined as hirsutism (FerrimanGallwey score>8), or minor signs such as acne, seboborrhea; and

- Criteria for polycystic ovary by ultrasound examination (minimum of 12 follicles with 2-9 mm diameters in each ovary and/or increasing ovarian volume with a minimum size of $10 \mathrm{~mm}^{3}$ ).

PCOS patients fulfilling the diagnostic criteria were then classified to their corresponding PCOS phenotypes $(\mathrm{A} / \mathrm{B} / \mathrm{C} / \mathrm{D})$. Informed consent was obtained from all women, and approval from the human ethics committee was obtained.

The control group consisted of women without endometriosis, cysts, or other ovarian gynecological disorders; had regular menstrual cycles (26-35 days); did not have endocrine abnormalities (prolactin, FSH, and basal estradiol at normal levels and not in a state of hyperandrogenism); and had morphologically normal 
ovaries according to ultrasound. Clinical data were collected from the participants including demographic details such as age, presenting complains (hirsutism, menstrual history, and symptoms of androgen excess).

Clinical history included complaint of oligomenorrhea, hirsutism and examination included FG score and BMI. Oligomenorrhea was taken as fewer than eight menstrual cycles during the previous 12 months or menstrual interval of more than 35 days. Clinical hyperandrogenism was defined as Ferriman-Gallwey score of $>8$. PCOM was diagnosed when either ovary on ultrasound had more than 12 follicles with a diameter of $2-9 \mathrm{~mm}$ or when ovarian volume was more than $10 \mathrm{cucm}$.

A detailed general examination was conducted for identification of acne, hirsutism and acanthosis nigricans. Blood sample was withdrawn and put in plain vials on day 2-3 of menses or after withdrawal bleeding. Samples were then centrifuged at $3000 \mathrm{rpm}$ in centrifugation machine at the biochemistry laboratory for serum analysis. AMH levels were run on ELISA kit by immune concept bio-detect which consisted of 96 wells with six standards. Analysis was done using a competitive enzyme immunoassay technique utilizing a monoclonal antiAMH antibody and an AMH-HRP conjugate on an antiAMH-coated plate. The minimum detection level for the kit was $0.025 \mathrm{ng} / \mathrm{ml}$. On the other sample, hormonal assays for serum TSH, FSH, LH, estradiol was performed using chemiluminescence immunoassay. Trans abdominal ultrasound was performed for all the women to detect PCOM.

Results of the above laboratory investigations and imaging studies were recorded along with clinical data of the patient in a proforma

Patients were classified as PCOS cases and controls according to the inclusion and exclusion criteria mentioned above

Data were entered in MS excel spreadsheet, and statistical analysis was done using statistical package for social sciences (SPSS) version 21.0.

The values are expressed in mean \pm SD. Statistical significance of differences between PCOS and non PCOS were carried out by unpaired t-test or non-parametric test. Multivariate analysis was done by using regression analysis and Pearson's coefficient of correlation. The level of significance was considered as $\mathrm{p}<0.05$.

Receiver operating characteristic curve was used to find out the cut off of AMH for predicting PCOS. Diagnostic test was used to find out the sensitivity, specificity, negative predictive value (NPV) and positive predictive value (PPV) of AMH for diagnosing PCOS.

\section{RESULTS}

In this study 50 patients of age group 18 to 39 years with PCOS (based on Rotterdam criteria) and 50 patients of same age group as controls (non PCOS) were selected from OPD of obstetrics and gynecology department of LLRM medical college Meerut during the period May 2018 to June 2019. In this study among PCOS cases oligomenorrhea was found in $80 \%$ of patients, hirsutism in $48 \%$ of patients and polycystic ovaries on ultrasound was found in $88 \%$ of PCOS cases but none of controls had PCOM as it was the exclusion criteria for controls.

Age group was comparable among the PCOS cases and NON PCOS controls. Age range for both groups was from 18 to 39 years with majority of patients in age group of 21-30 years (cases $70 \%$ and controls $58 \%$ ). The mean age of PCOS cases and controls was 26.26 and 30.48 years, respectively, and the difference was not statistically significant. The mean BMI of PCOS cases was comparable with the control group. Mean BMI of cases was $25.29 \mathrm{~kg} / \mathrm{m}^{2}$ and controls was $24.98 \mathrm{~kg} / \mathrm{m}^{2}$ and there was no statistically significant difference between the two groups $(\mathrm{p}=0.39)$, majority of PCOS cases $(56 \%)$ and controls $(54 \%)$ were overweight $(\mathrm{p}=1.278)$.

Regarding different phenotypes of PCOS most frequent phenotype found in this study was phenotype A $(48 \%)$ i.e., 24 out of 50 PCOS patients were having $\mathrm{OA}+\mathrm{HA}+\mathrm{PCOM}$ i.e., having all the three features of Rotterdam criteria. Highest mean level of AMH found in phenotype A (8.44 ng/ml).

The mean level of serum FSH in PCOS cases was 6.6704 and in non PCOS group was 7.483, ( $\mathrm{p}$ value $=0.09$ ) this shows no statistically significant difference in cases and controls. The mean level of serum (LH)luteinizing hormone in PCOS cases was 12.9204 and in non PCOS group was 8.735 , ( $\mathrm{p}$ value $=0.000001$ ) which shows statistically significant difference of LH levels in cases and controls in this study. Mean level of AMH in PCOS cases and controls was 7.1096 and 2.423 respectively, serum level of AMH in women with PCOS were two to three times higher than the levels in women without PCOS $(\mathrm{p}<0.05)$ which is statistically significant.

On applying receiver operating characteristic curve (ROC curve) analysis, area under the curve was 0.98 [95\% CI 0.929 to 0.998$)$; $\mathrm{p}$ value $<0.0001]$. In present study the maximum diagnostic potency of AMH alone for PCOS was at cut-off of $4.22 \mathrm{ng} / \mathrm{ml}$ with sensitivity of $92 \%(95 \%$ CI 80.8 to 97.8 ) and specificity of $100 \%$ (95\% CI 92.9 to 100).

The positive predictive value is $100 \%$ (95\% CI 92.3 to $100.0)$ and negative predictive value is $92.6 \%$ (95\% CI 82.1 to 97.9 ) (Figure 1). 
Table 2: AMH levels of the four groups based on PCOS related phenotypes.

\begin{tabular}{|llllll|}
\hline Phenotypes & OA & HA & PCOM & Frequency $(\%)$ & Mean AMH $(\mathrm{ng} / \mathrm{ml})$ \\
\hline A & + & + & + & 48 & 8.44 \\
\hline B & + & + & - & 12 & 6.28 \\
\hline C & - & + & + & 4 & 5.84 \\
\hline D & + & - & + & 36 & 5.72 \\
\hline
\end{tabular}

Table 3: AMH as a diagnostic tool for PCOS in various studies.

\begin{tabular}{|llllll|}
\hline References & Year & Study design & No. of patients & $\begin{array}{l}\text { Mean AMH } \\
\text { values (ng/ml) }\end{array}$ & $\begin{array}{l}\text { Cut off value of } \\
\text { AMH }(\mathbf{n g} / \mathbf{m l})\end{array}$ \\
\hline Siow et al & 2005 & Prospective & 31 & 4.1 & \\
\hline Pigny et al & 2006 & Prospective & 73 & 11.42 & 8.4 \\
\hline Wachs et al & 2007 & Prospective & 16 & 7.2 & \\
\hline Dewailly et al & 2010 & Retrospective & 270 & 7.88 & 4.90 \\
\hline Hart et al & 2010 & Prospective & 64 & 3.08 & 4.20 \\
\hline Park et al & 2010 & Prospective & 153 & 5.28 & \\
\hline Li et al & 2010 & Retrospective & 47 & 9.85 & 8 \\
\hline Skalba et al & 2011 & Retrospective & 87 & 10.2 & \\
\hline Woo et al & 2012 & Retrospective & 140 & 11.58 & 7.82 \\
\hline Sahmay et al & 2013 & Retrospective & 419 & 7.34 & 3.94 \\
\hline Wiweko et al & 2014 & Case control & 71 & 9.50 & 4.45 \\
\hline Saxena & 2017 & Prospective case control & 90 & 4.32 & 3.44 \\
\hline Present study & 2019 & Prospective case control & 100 & 7.1096 & 4.22 \\
\hline
\end{tabular}

Table 4: Differences of basal hormone levels, age and BMI between PCOS and non PCOS patients.

\begin{tabular}{|llll|}
\hline Variables & PCOS & Non PCOS & P value \\
\hline Age & $26.26 \pm 4.44$ & $30.48 \pm 2.76$ & 1.278 \\
\hline BMI & $25.29 \pm 1.92$ & $24.98 \pm 1.64$ & 0.39 \\
\hline AMH & $7.10 \pm 3.73$ & $2.42 \pm 0.79$ & $<0.05$ \\
\hline FSH & $6.67 \pm 2.04$ & $7.48 \pm 2.70$ & 0.09 \\
\hline LH & $12.92 \pm 5.68$ & $8.73 \pm 3.19$ & $<0.05$ \\
\hline
\end{tabular}

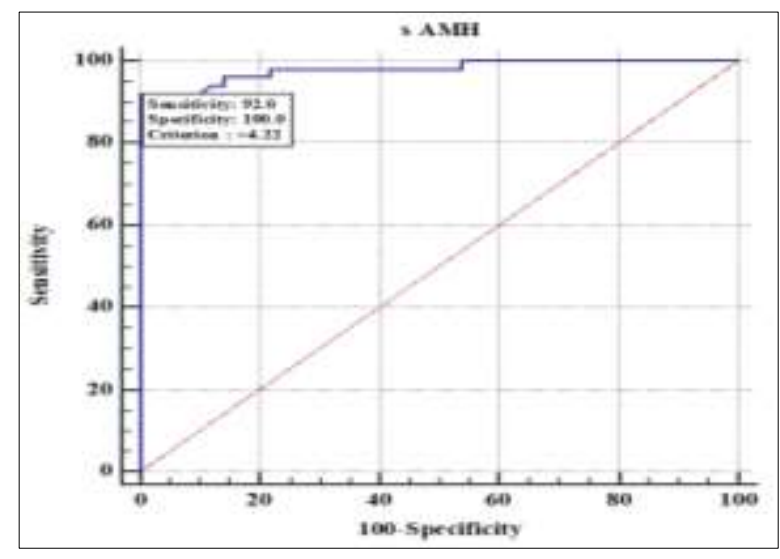

Figure 1: Receiver operator characteristic curve.

\section{DISCUSSION}

The use of ultrasound to diagnose PCOS can be problematic, the interpretation of ultrasound results may demand subjective judgments and is subjected to interobserver variability. Moreover, the vast majority of the target population are teenagers and women of reproductive age, and they may be unavailable for transvaginal ultrasound evaluation because of their virginal status or obesity. In addition to unclear diagnostic criteria, the presence of different phenotypes with PCOS further complicates the diagnosis.

The technical advances in imaging have led to an artificial increase in PCOM resulting in confusion over its use as diagnostic criteria.

The diagnosis of PCOS requires an objectives and quantitative criteria to help clinicians to diagnose and treat patients suffering from this complex endocrine disorder.

The present study investigated the serum level of AMH as a diagnostic marker for PCOS and showed that the serum level of AMH in women with PCOS were two to 
three times higher than the levels in women without PCOS $(p<0.05)$. Authors found significantly higher serum AMH level in PCOS women compared to the controls. This finding has consistently been reported in numerous studies.

Age group was comparable among the PCOS cases and NON PCOS controls in this study, this finding correlates with the study of Saxena et al, in which no statistically significant difference was found between age groups of PCOS cases and control groups however different result was found in study of Wiweko et al, Rousseau et al, Johnstone et al, in which the average age of PCOS patients was significantly younger than non PCOS patients $(p=0.008)$, they concluded that the proportion of women with PCO decreased with age. ${ }^{11-14}$

The mean BMI of PCOS patients was comparable with the control group, this was in agreement with previous studies of Wiweko et al, Saxena et al, Sezai et al in which BMI was comparable between both the groups and no significant differences was found among the two groups.

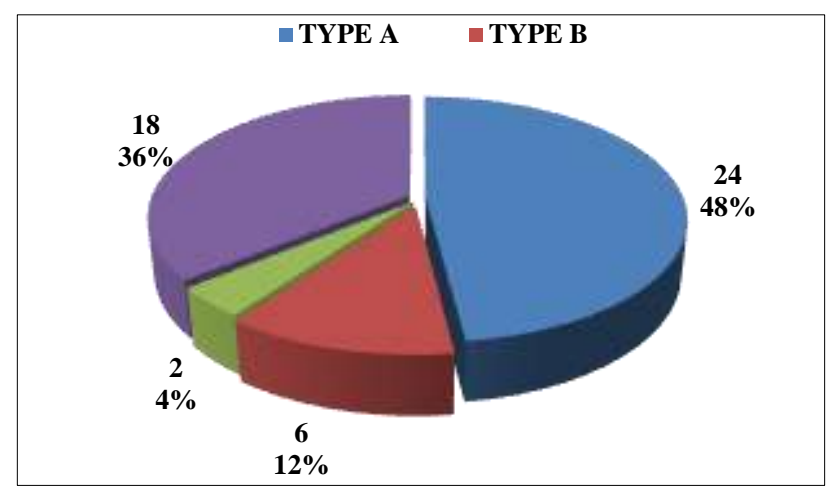

Figure 2: Frequency of different phenotypes.

Most frequent phenotype in this study was phenotype A (Figure 2).

However, in Wiweko et al study the most frequent PCOS phenotype was phenotype $\mathrm{D}(\mathrm{OA}+\mathrm{PCOM})$ which was the next most frequent PCOS phenotype after phenotype $\mathrm{A}$ in this study. Phenotype $\mathrm{C}$ was the most frequent phenotype observed in Bozdag et al, study however their study showed the highest mean AMH level in phenotype A (patients having all the three features of syndrome). ${ }^{15}$ Highest level of serum AMH (11.1 ng/ml) was also found in phenotype A in Wiweko study. This is in line with this present study in which highest mean level of $\mathrm{AMH}$ found in phenotype A $(8.44 \mathrm{ng} / \mathrm{ml})$, this can correlates with severity of disease i.e., patients having all the three features of syndrome $(\mathrm{OA}+\mathrm{HA}+\mathrm{PCOM})$ have highest $\mathrm{AMH}$ level, this shows that the concentrations of serum AMH correlate with the severity of symptoms (Table 2).

The same result was also found in an Indian study of Saxena in which highest level of serum AMH was found in phenotype A.
Several studies have reported increased serum AMH levels in women with PCOS compared with controls, and the role of $\mathrm{AMH}$ as a diagnostic marker has been evaluated in previous studies (Table 3 ).

However, both mean serum AMH levels and suggested cut-off values for AMH were inconsistent among the studies, probably because of differences in sample size, sample selection criteria, and specified PCOS phenotypes among the studies. Such difference in the cut off value might be because of different types of AMH kits being used. ELISA in these studies was by diagnostic system laboratories (DSL) whereas authors used kit from immune concept bio detect.

The results of the present study are compatible with previous results only in terms of an elevated serum AMH level in women with PCOS.

Pigny et al evaluated serum AMH levels in the diagnosis of PCOS; they reported a satisfactory specificity of $92 \%$ but a low sensitivity of $67 \%$ with an AMH cutoff of 8.4 $\mathrm{ng} / \mathrm{mL}(60 \mathrm{lmol} / \mathrm{L})$ and a mean serum AMH of 11.42 ng/mL (81.6 lmol/L). ${ }^{16}$ Both the mean AMH and AMH cut-off values were higher than the values in the present study, possibly due to their small patient population.

$\mathrm{Li}$ et al, reported that serum AMH levels were elevated in adolescent young adult Chinese women with PCOS, but the serum AMH measurements offered a relatively poor diagnostic power, with a sensitivity of $61.7 \%$ and a specificity of $70 \%$ at a cut-off of $8 \mathrm{ng} / \mathrm{mL} .{ }^{17}$ They suggested that the low specificity and sensitivity in their study was attributable to the lower prevalence of hyperandrogenism, obesity, and insulin resistance in their cohort owing to racial differences.

Hart et al, found the most effective cut-off value of AMH to be $4.2 \mathrm{ng} / \mathrm{mL}(30 \mathrm{lmol} / \mathrm{L})$, which is close to this study findings. Pellat et al, reported that $\mathrm{AMH}$ production increases approximately 75 times higher in each polycystic ovarian granulosa cell. ${ }^{18,19}$ This finding is also supported by Catteau-Jonard et al who found increased mRNA expression of AMH in ovarian granulosa cells. Weerakiet's et al stated that AMH plasma levels can be a marker of the degree to which folliculogenesis is impaired in patients with PCOS. ${ }^{20}$ A study by Dewailly et al indicated that AMH may also be used as a surrogate marker of classical hyperandrogenism. ${ }^{21}$ Several other studies emphasize that the concentration of $\mathrm{AMH}$ is associated with the severity of morphological and hormonal changes in PCOS patients.

On applying receiver operating characteristic curve (ROC curve) analysis, area under the curve was 0.98 (95\% CI 0.929 to 0.998 ); $\mathrm{p}$ value $<0.0001$ ) (Figure 1). In present study the maximum diagnostic potency of AMH alone for PCOS was at cut-off of $4.22 \mathrm{ng} / \mathrm{ml}$ with sensitivity of $92 \%$ (95\% CI 80.8 to 97.8$)$ and specificity of $100 \%(95 \%$ CI 92.9 to 100$)$. 
The positive predictive value is $100 \%$ (95\% CI 92.3 to $100.0)$ and negative predictive value is $92.6 \%$ (95\% CI 82.1 to 97.9$)$.

This result of ROC curve was similar to study of an Indian study Saxena in which area under the curve is 0.778 (95\% CI 0.678 to 0.859 : $p$ value <0.0001). Maximum diagnostic potency of AMH was at cut off of $3.44 \mathrm{ng} / \mathrm{ml}$ in Saxena study with a sensitivity of $77.78 \%$ and specificity of $68.89 \%$.

Similar cut-off of AMH of $3.34 \mathrm{ng} / \mathrm{ml}$ with higher sensitivity of $98 \%$ and specificity of $93 \%$ was reported by an Indian study of Saikumar et al this was also in congruence with previous studies. ${ }^{22}$

Woo et al and Lin et al reported low sensitivity and specificity than the present study but at higher cut-off level of $7.82 \mathrm{ng} / \mathrm{ml}$ and $7.3 \mathrm{ng} / \mathrm{ml}$ respectively. ${ }^{23,24}$

Dewailly et al observed a higher sensitivity and specificity of $92 \%$ and $97 \%$ respectively at a cut-off of $4.9 \mathrm{ng} / \mathrm{ml} .^{21}$ However, Homburg et al reported high specificity $98.2 \%$ but low sensitivity $60 \%$ of AMH at cutoff of $6.7 \mathrm{ng} / \mathrm{ml}$.

Based on these findings, If at sometimes, ultrasound cannot provide accurate data, the levels of AMH may be used to replace the number of follicles as a diagnostic criterion. Serum AMH measurement is very valuable in the diagnosis of PCOS women. The serum AMH level in women with hyperandrogenism or oligo-anovulation could indicate the diagnosis of PCOS when reliable ultrasonography data are not available or when typical clinical and laboratory findings are not available. The serum AMH level is a new and useful diagnostic tool in PCOS diagnosis.

Though no single cut-off of AMH is diagnostic but still it is a promising diagnostic tool for PCOS as AMH is not affected by the day of menses or OCP intake, hence can be used as a biological, objective, quantitative marker for diagnosing PCOS. So, in future, more studies should be undertaken to validate its role as diagnostic tool for PCOS.

Further research will be needed to evaluate the relationship of AMH levels with lipid profiles and metabolic syndromes and to evaluate $\mathrm{AMH}$ as a tool for monitoring the success of PCOS treatment.

\section{Funding: No funding sources}

Conflict of interest: None declared

Ethical approval: The study was approved by the Institutional Ethics Committee

\section{REFERENCES}

1. Sahmay S, Atakul N, Aydogan B, Aydın Y, Imamoglu M, Seyisoglu H. Elevated serum levels of
anti-Müllerian hormone can be introduced as a new diagnostic marker for polycystic ovary syndrome. Acta Obstet Gynecol Scand. 2013;92(12):1369-74.

2. Bozdag G, Mumusoglu S, Coskun ZY, Yarali H, Yildiz BO. Anti-müllerian hormone as a diagnostic tool for PCOS under different diagnostic criteria in an unselected population. Reprod Biomed. 2019;39(3):522-9.

3. Umland EM, Weinstein LC, Buchanan EM. Menstruation-related disorders. In: DiPiro JT, Talbert RL, Yee GC, et al. Editors. Pharmacotherapy: a pathophysiologic approach. $8^{\text {th }}$ ed. New York: McGraw-Hill; 2011:1393.

4. Lin LH, Baracat MC, Maciel GA, Soares JM, Baracat EC. Androgen receptor gene polymorphism and polycystic ovary syndrome. Int $\mathbf{J}$ Gynaecol Obstet. 2013;120:115-118.

5. Aubuchon M, Legro RS. Polycystic ovary syndrome: Current infertility management. Clin Obstet Gynecol. 2011;54(4):675-84.

6. Broekmans FJ, de Ziegler D, Howles CM, Gougeon A, Trew G, Olivennes F. The antral follicle count: practical recommendations for better standardization. Fertil Steril. 2010;94(3):1044-51.

7. Duijkers IJ, Klipping C. Polycystic ovaries, as defined by the 2003 Rotterdam consensus criteria, are found to be very common in young healthy women. Gynecol Endocrinol. 2010;26(3):152-60.

8. Johnstone EB, Rosen MP, Neril R, Trevithick D, Sternfeld B, Murphy R, et al. The polycystic ovary post-Rotterdam: a common, age dependent finding in ovulatory women without metabolic Bibliography 85 significance. J Clin Endocrinol Metabol. 2010;95(11):4965-72.

9. Kristensen SL, Ramlau-Hansen CH, Ernst E, Olsen SF, Bonde JP, Vested A, et al. A very large proportion of young Danish women have polycystic ovaries: is a revision of the Rotterdam criteria needed?. Human Reprod. 2010;25(12):3117-22.

10. Bako AU, Morad S, Atiomo WA. Polycystic ovary syndrome: an overview. Rev Gynaecol Pract. 2005;5(2):115-22.

11. Saxena U, Ramani M, Singh P. Role of AMH as diagnostic tool for polycystic ovarian syndrome. The J Obstet Gynecol India. 2018;68(2):117-22.

12. Wiweko B, Maidarti M, Priangga MD, Shafira N, Fernando D, Sumapraja K, et al. Anti-mullerian hormone as a diagnostic and prognostic tool for PCOS patients. J Assisted Reprod Genet. 2014;31(10):1311-6.

13. Johnstone EB, Rousseau JA, Lamb JD, Huddleston HG, Cedars MI. Age bias in polycystic ovary syndrome (PCOS) diagnostic criteria limits diagnosis among those at greatest cardiovascular risk. Fertil Steril. 2009;92(3):S38.

14. Johnstone EB, Rosen MP, Neril R, Trevithick D, Sternfeld B, Murphy R, et al. The polycystic ovary post-rotterdam: a common, age-dependent finding in ovulatory women without metabolic significance. J Clin Endocrinol Metab. 2010;95(11):4965-72. 
15. Yildiz BO, Bozdag G, Yapici Z, Esinler I, Yarali H. Prevalence, phenotype and cardiometabolic risk of polycystic ovary syndrome under different diagnostic criteria. Human Reprod. 2012;27(10):3067-73.

16. Pigny P, Jonard S, Robert Y, Dewailly D. Serum anti-mullerian hormone as a surrogate for antral follicle count for definition of the polycystic ovary syndrome. J Clin Endocrinol Metabol. 2006;91(3):941-5.

17. Li Y, Ma Y, Chen X, Wang W, Li Y, Zhang Q, et al. Different diagnostic power of anti-mullerian hormone in evaluating women with polycystic ovaries with and without hyperandrogenism. J Assist Reprod Genet. 2012;29(10):1147-51.

18. Hart R, Doherty DA, Norman RJ, Franks S, Dickinson JE, Hickey M, et al. Serum anti-mullerian hormone (AMH) levels are elevated in adolescent girls with polycystic ovaries and the polycystic ovarian syndrome (PCOS). Fertil Steril. 2010;94:1118-21.

19. Pellatt L, Hanna L, Brincat M, Galea R, Brain H, Whitehead S, et al. Granulosa cell production of antimullerian hormone is increased in polycystic ovaries. J Clin Endocrinol Metab. 2007;92(1):240-5.

20. Weerakiet S, Lertvikool S, Tingthanatikul Y, Wansumrith S, Leelaphiwat S, Jultanmas R. Ovarian reserve in women with polycystic ovary syndrome who underwent laparoscopic ovarian drilling. Gynecol Endocrinol. 2007;23(8):455-60.
21. Dewailly D, Gronier H, Poncelet E, Robin G, Leroy M, Pigny P, et al. Diagnosis of polycystic ovary syndrome (PCOS): revisiting the threshold values of follicle count on ultrasound and of the serum AMH level for the definition of polycystic ovaries. Hum Reprod. 2011;26(11):3123-9.

22. Saikumar P, SelVi VK, Prabhu K, VenKateSh P, KriShna P. Anti-mullerian hormone: a potential marker for recruited non growing follicle of ovarian pool in women with polycystic ovarian syndrome. J Clin Diagn Res. 2013;7(9):1866-9.

23. Woo H, Kim KH, Rhee EJ, Park H, Lee MK. Differences of the association of anti-Mullerian hormone with clinical or biochemical characteristics between women with and without polycystic ovary syndrome. Endocr J. 2012;59(9):781-90.

24. Lin Y, Chiu WC, Wu CH, Tzeng CR, Hsu CS, Hsu MI. Antimullerian hormone and polycystic ovary syndrome. Fertil Steril. 2011;96:230-5.

Cite this article as: Singh S, Firdaus A, Chaudhary $\mathrm{R}$, Dhama V. Role of anti-mullerian hormone as a diagnostic tool for polycystic ovary syndrome. Int $\mathbf{J}$ Reprod Contracept Obstet Gynecol 2020;9:3730-6. 